\title{
Evaluating photoneutron dose equivalents for lung cancer using PMMA phantoms undergoing 15 MV IMRT
}

\author{
Hai-Bing $\mathrm{Xu}^{\mathrm{a}}$, An-Ding Dong ${ }^{\mathrm{a}}$, Mu-Tai Liu ${ }^{\mathrm{b}}$, Hsien-Chun Tseng ${ }^{\mathrm{c}, \mathrm{d}}$, Chien-Yi Chen ${ }^{\mathrm{c}, \mathrm{e}, 1, *}$ and \\ Sung-Yi Tsai ${ }^{\mathrm{a}, 1, *}$ \\ ${ }^{a}$ Department of Medical Imaging, Jiangsu Vocational College of Medicine, Yancheng, Jiangsu, China \\ ${ }^{\mathrm{b}}$ Department of Radiation Oncology, Changhua Christian Hospital, Changhua, Taiwan \\ ${ }^{\mathrm{c}}$ Department of Radiation Oncology, Chung Shan Medical University Hospital, Chung Shan Medical \\ University, Taichung, Taiwan \\ ${ }^{\mathrm{d}}$ School of Medicine, Chung Shan Medical University, Taichung, Taiwan \\ ${ }^{\mathrm{e}}$ Department of Medical Imaging and Radiological Sciences, Chung Shan Medical University, Taichung, \\ Taiwan
}

\begin{abstract}
.
BACKGROUND: According to statistics of the Ministry of Health and Welfare in 2017, the second leading cause of death in Taiwan was lung cancer.

OBJECTIVE: Routine treatment planning does not consider photoneutron dose equivalent (PNDE) of patient induced secondary radiation resulting from primary exposure of lung cancer. However, such treatment is potentially important for improving estimates of health risks.

METHODS: This study used 10,30,50,70, and $90 \mathrm{~kg}$ of polymethylmethacrylate (PMMA) phantoms as patient to measure PNDE varying anatomical area during lung cancer of intensity modulated radiotherapy (IMRT) treatment. Paired thermoluminescent dosimeters (TLD-600 and 700) were calibrated using university reactor neutrons. TLDs were inserted into phantom which was closely corresponded of the represented tissues or organs.

RESULTS: Neutron doses (ND) of organ or tissue $\left(N D_{T}\right)$ were determined in these phantoms using paired TLDs approach. The risks of incurring fatal secondary malignancies, maximum statistical and total errors were estimated. Evaluated PNDE ranged from $0.80 \pm 0.12$ to $0.56 \pm 0.08 \mathrm{mSv} / \mathrm{Gy}$ for these phantoms.

CONCLUSION: The estimated $N D_{T}$ decreased with increasing distance that is from the central axis. Evaluated PNDE and $N D_{\text {skin }}$ for these phantoms were discussed. This investigation also identified secondary risks associated with PNDE relating to radiation protection.
\end{abstract}

Keywords: Photoneutron dose equivalent, lung cancer, phantom, thermoluminescence dosimeter, risk assessment

\section{Introduction}

Medical electron linear accelerators (linac) operating above $10 \mathrm{MV}$ produce high-energy bremsstrahlung

\footnotetext{
${ }^{1}$ These authors contributed equally as corresponding authors.

${ }^{*}$ Corresponding authors: Chien-Yi Chen, Department of Medical Imaging and Radiological Sciences, Chung Shan Medical University, Taichung, Zhejiang 40201, China. E-mail: chien.ccy@gmail.com. Sung-Yi Tsai, Department of Medical Imaging, Jiangsu Vocational College of Medicine, Yancheng, Jiangsu, China. E-mail: 2718840016@qq.com.
} 
photon, then induce neutrons via $(\mathrm{x}, \mathrm{yn})$ reactions [1-3]. These reactions occur at the accelerator target (tungsten, iron), flattening filters, shielding materials, collimator jaws (inside the linac head), and in the patient body [1-4]. The linac treatment room contains a mixed radiation field produced by the induced neutrons and photons. The medical linac, Siemens Primus (Siemens AG, Munich, Germany) at the Radiation Oncology Department of Changhua Christian Hospital (CCH) which could provide energies that accelerated 10 and $15 \mathrm{MV}$ voltages. The linac is designed of intensity modulated radiotherapy (IMRT). The fast neutron flux $\left(\Phi_{f}\right)$ for $15 \mathrm{MV}$ of linac was $1.75 \times 10^{4}\left(\mathrm{n} / \mathrm{cm}^{2} \mathrm{~s}\right) / \mathrm{Gy}-\mathrm{X}$, as well as flux of thermal neutron $\left(\Phi_{t h}\right)$ was $1.26 \times 10^{5}\left(\mathrm{n} / \mathrm{cm}^{2} \mathrm{~s}\right) / \mathrm{Gy}-\mathrm{X}$ at SSD of $100 \mathrm{~cm}$, respectively [5]. Patient thus was exposed to significant undesirable radiations comprising photons and neutrons leakage (both direct and scattered components) [1]. Knowledge of the undesirable photoneutron dose equivalent (PNDE) in patients of different body weights is necessary. These data were necessary to determine safety and improved treatment plan of lung cancer in patients [1]. According to ICRP 60, the radiation weighting factor $\left(W_{R}\right)$ for neutrons ranging from 5 to $20 \mathrm{MeV}$ is quite high, and one would expect significant PNDE to the critical organs, like gonads and lung [5-7]. A series of measurements and calculations were carried out to determine the PNDE and neutron dose of organ or tissue $\left(N D_{T}\right)$ in the patient undergoing $15 \mathrm{MV}$ linac treatments for lung cancer. The authors are aware of limited studies assessing the secondary ND that results from differences in patient weight from step-and-shot IMRT [1]. The experiment described here is to use polymethylmethacrylate (PMMA) phantoms because of different body-weight with the same anthropometric-shaped skeletons as patient surrogates to assess the PNDE of various anatomical areas. Paired TLDs dosimeters (TLD-600 and 700) (Harshaw Chemical Company, Solon, OH, USA) were arranged and inserted. Simple equation could be evaluated PNDE during phantoms' lung cancer. High $N D_{\text {lung }}$ values were recorded up to $1.02 \pm 0.13 \mathrm{mSv} / \mathrm{Gy}$ of Rando. This study is recommended to prevent extra ND to patients and could be satisfied the ALARA [7].

\section{Materials and methods}

\subsection{Polymethylmethacrylate (PMMA) phantom measurement}

Lung treatment plans of IMRT have been developed, delivered to anthropomorphic Rando phantom. This was made of Radiology Support Devices, Long Beach, Canada. Treatment planes of these phantoms were reviewed by professional medical doctor (Mu-Tai Liu, MD) and senior radiotherapist (Sing-Sheng Huang) [7-11]. To calculate the PNDE associated with IMRT treatment, this study conducted paired TLDs measurement in Rando and PMMA phantoms. The Rando phantom was $175 \mathrm{~cm}$ and $73.5 \mathrm{~kg}$. This phantom was comprised 35 axial segments, those contained simulated human skeleton as well as lung soft tissue. Rando is suitable for IMRT radiation measurements [4]. The phantom was sliced at $2.5 \mathrm{~cm}$ intervals and holes were drilled in each slice for TLD-600 insertions. Paired TLDs were loaded into each slice of phantoms as well as can use to directly assess PNDE and secondary radiation to phantoms.

This study developed five phantoms using anthropometric skeletons made of PMMA and epoxyresin to simulate average body-weights of $90,70,50,30$ and $10 \mathrm{~kg}$ [12]. The PMMA phantom was designed as published [9]. PMMA and epoxy-resin were chosen as the neutron and photon transport properties. Density were formulated as follows: skeleton-cortical-bone, 1.486; skin, 1.105; and lungtissue $0.296 \mathrm{~g} / \mathrm{cm}^{3}$ of PMMA, respectively $[9,12]$. The PMMA phantoms were made of the human design; contained 31 slices. Those were represented the head, neck, torso and abdomen. These were without two arms and legs [11,12]. Each slice was drilled with an $11 \mathrm{~mm}$ diameter hole to enable 
Table 1

Rando, polymethylmethacrylate (PMMA) phantoms' dimension and physical properties

\begin{tabular}{lcclllr}
\hline \multicolumn{1}{c}{ Phantom } & Rando & \multicolumn{5}{c}{ Polymethylmethacrylate (PMMA) } \\
\hline Weight $(\mathrm{kg})^{1}$ & 70 & 10 & 30 & 50 & 70 & 90 \\
Height $(\mathrm{cm})^{2}$ & 94.5 & 50 & 78 & 84 & 93 & 112 \\
Weight $(\mathrm{kg})^{2}$ & 34.5 & 6.75 & 19.0 & 31.5 & 44.1 & 57 \\
Slices & 35 & 31 & 31 & 31 & 31 & 31 \\
cm/slices & 2.5 & 1.6 & 2.3 & 2.7 & 3.0 & 3.6 \\
\hline
\end{tabular}

insertion of the TLDs into organs. The dimensions and physical properties of theses phantoms is listed in Table 1.

\subsection{Paired TLDs}

For subtracting the signal (SN) contributed by photons from total SNs of TLD-600, and 700, all neutron measurements were performed using paired TLDs approach composed with a batch of 88 high sensitivity $3.2 \times 3.2 \times 0.89 \mathrm{~mm}^{3}$ dosimeters [10]. TLD-600 comprises $95.6 \%{ }^{6} \mathrm{Li}$, and $4.4 \%{ }^{7} \mathrm{Li}$. TLD-700 is $99.7 \%$ ${ }^{7} \mathrm{Li} .{ }^{6} \mathrm{Li}$ and ${ }^{7} \mathrm{Li}$ of these TLDs could provide different responses to neutron reactions of the ${ }^{6} \mathrm{Li}(\mathrm{n}, \alpha)^{3} \mathrm{H}$ nuclear reaction [9]. Before irradiation, paired TLDs were annealed by heating to $400^{\circ} \mathrm{C}(1 \mathrm{hr})$ and $200^{\circ} \mathrm{C}$ $(2 \mathrm{hr}$ ) in furnace 47900 . Then TLDs were cooled to normal temperature before use [10]. Following IMRT exposure, a fully automated Harshaw 3500 reader made of Bicron NE Solon, $\mathrm{OH}$ was used to analyzed these TLDs. The readout SN involved a two steps, (A) heating to $300^{\circ} \mathrm{C}$ at speed of $10^{\circ} \mathrm{C}$ per second, holding for a further 1 second, (B) heating to $50^{\circ} \mathrm{C}$ (1 second) [10].

\subsection{Calibrated paired TLDs}

The ND calibrations were performed at the boron neutron capture therapy (BNCT) beams of National Tsing Hua University (NTHU). Using BNCT beams are $3.10 \times 10^{7} \mathrm{n} / \mathrm{cm}^{2} \mathrm{~s}\left(\Phi_{f}\right)$, as well as $1.31 \times$ $10^{8} \mathrm{n} / \mathrm{cm}^{2} \mathrm{~s}\left(\Phi_{t h}\right)$ measured at the exit of the beam for reactor power of $1 \mathrm{MW}$ [9]. Additionally, the $\Phi_{t h}$ kerma factor of TLD-600s was calibrated as $1.40 \times 10^{-10} \mathrm{mGy} /\left(\mathrm{n} / \mathrm{cm}^{2} \mathrm{~s}\right)$ by Monte Carlo simulation [9]. For paired TLDs' photon dose calibration, linearity calibration was carried by Siemens Primus of CCH' $6 \mathrm{MV}$ therapeutic unit [6]. This machine typically provides a dose of $400 \mathrm{MU}$ (monitor unit)/min, corresponding to $3.3 \mathrm{~Gy} / \mathrm{min}$. For $10 \mathrm{~cm} \times 10 \mathrm{~cm}$ field size, $1 \mathrm{MU}$ equals approximately $1 \mathrm{cGy}$ at SSD $100 \mathrm{~cm}$. Paired TLDs were arranged in 10-cm-diameter methacrylate boxes. An ionization chamber (IC) like pencil-shaped, Wellhofer IC-69, CT Probes Models 350407 made by Nuclear Associates Victoreen, Inc., containing $0.6 \mathrm{~cm}^{3}$ was used to measure X-ray radiation. The IC was connected by way of $0.9 \mathrm{~m}$ low-noise cable to a SN digitizing preamplifier. Due to subtracting photon response, ND in these phantoms is calculated as

$$
\begin{aligned}
& S N_{600}^{n+\gamma}=S N_{600}^{n}+S N_{600}^{\gamma} \\
& S N_{700}^{n+\gamma}=S N_{700}^{n}+S N_{700}^{\gamma}
\end{aligned}
$$

Where $S N_{600}^{n+\gamma}$ is denoted as total TLD-600 SN. $S N_{600}^{n}$ is net neutron SN. $S N_{600}^{\gamma}$ is $\gamma$-ray contribution to TLD-600 SN. $S N_{700}^{n+\gamma}$ is denoted as total TLD-700 SN. $S N_{700}^{n}$ and $S N_{700}^{\gamma}$ are the respective contribution to TLD-700 SN of neutron and $\gamma$-ray.

$$
S N_{600}^{n}=S N_{600}^{n+\gamma} \times C F_{600}^{n}
$$




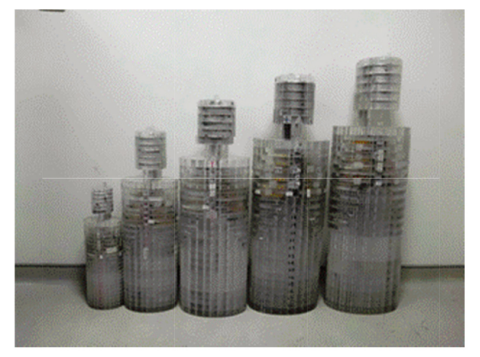

(a)

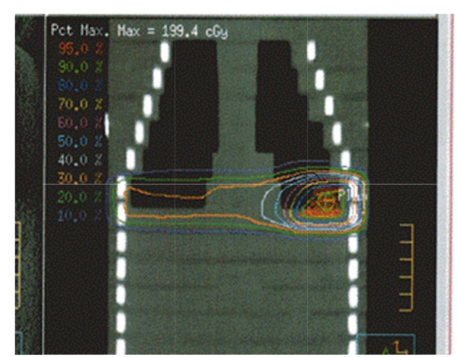

(b)

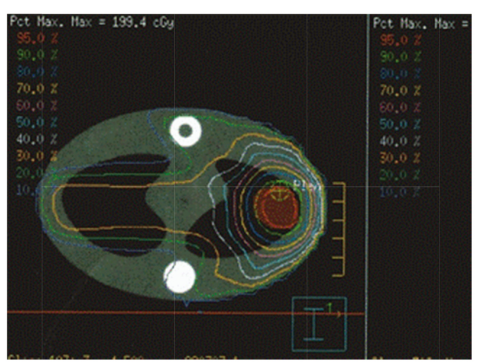

(c)

Fig. 1. Use polymethylmethacrylate (PMMA) phantoms as patient surrogates (a) outer appearances (b) from 9th to 20th scout view of $70 \mathrm{~kg}$ (c) 15 th CT slice of $70 \mathrm{~kg}$ phantom.

$C F_{600}^{n}$ is conversion factor (CF) of ND of TLD-600 calibrated by BNCT.

\subsection{Computed tomography (CT) scans and treatment planning for lung cancer}

Before each treatment, numerous measurements were conducted using inhomogeneous PMMA phantoms. This study obtained archival Computed tomography (CT) scan images of the phantoms at CCH. A $3 \mathrm{~cm} \times 3 \mathrm{~cm} \times 3 \mathrm{~cm}$ lung tumor, in the center of left lung at a depth of $5 \mathrm{~cm}$, is shown as Fig. 1a-c. Organ contours' CT images were transferred into Siemens Primus for IMRT. CT protocol scans of these phantoms for three-dimensional visualizations of the lung region were made by way of a multi-slice CT (MSCT) (high speed, General Electric). The settings of $\mathrm{mA}$ and $\mathrm{kV}$ of the CT scanner were varied according to the phantoms' weights. The lung block' size and place was obtained by way of the treatment planning system. After loading these TLDs into phantoms, three repeat scans were conducted over the specified area. All technique parameters as well as displayed quantities were recorded. The IMRT approaches were delivered to the phantoms with $200 \mathrm{cGy}$ prescribed to the planned target volume (PTV) to facilitate detection of the small doses outside the treatment field.

\subsection{Paired TLDs inserted into Rando and PMMA phantoms}

Calculation points are located in the lung and tumor center in the superior portion of the torso of these phantoms of radiotherapy $15 \mathrm{MV}$ linac during lung cancer treatment [1]. Forty-four groups, each containing 2 TLD-600 and 700s, were inserted into phantoms at the positions of slices 3-35 (Rando) and 3-31 (PMMA), respectively. For all measuring locations of NDs, two TLD-600s were irradiated together as well as average signal determined. Four group TLDs were attached to $15^{\text {th }}$ 's surface of the Rando' slices in order to measure the $N D_{\text {skin }}$. 
Table 2

44 ND measurement points, and the weighting factors $W_{T}$ recommended by ICRP 103

\begin{tabular}{|c|c|c|c|}
\hline Organ & Measurement points & ICRP 103 & Practical evaluation \\
\hline Brain & Brain & 0.01 & 0.01 \\
\hline Salivary & Salivary & 0.01 & 0.01 \\
\hline Thyroid & Thyroid & 0.04 & 0.04 \\
\hline \multicolumn{3}{|c|}{ Red bone marrow } & 0.12 \\
\hline & Clavicle vertebrae & & 0.04 \\
\hline & Thoracic vertebra & & 0.04 \\
\hline & Thighbone femur & & 0.04 \\
\hline Lung & Lung & 0.12 & 0.12 \\
\hline Skin & Skin & 0.01 & 0.01 \\
\hline Esophagus & Esophagus & 0.04 & 0.04 \\
\hline Breast & Breast & 0.12 & 0.12 \\
\hline \multirow[t]{5}{*}{ Bone surface } & & 0.01 & \\
\hline & Clavicle vertebrae & & 0.025 \\
\hline & Thoracic vertebra & & 0.025 \\
\hline & Thighbone & & 0.025 \\
\hline & Rib & & 0.025 \\
\hline Liver & Liver & 0.04 & 0.04 \\
\hline \multirow[t]{5}{*}{ Colon } & & 0.12 & \\
\hline & Ascending & & 0.03 \\
\hline & Transverse & & 0.03 \\
\hline & Descending & & 0.03 \\
\hline & Sigmoid flexure & & 0.03 \\
\hline Stomach & Stomach & 0.12 & 0.12 \\
\hline Bladder & Bladder & 0.04 & 0.04 \\
\hline Gonads & Testes & 0.08 & 0.08 \\
\hline \multirow[t]{8}{*}{ Remainder } & & 0.12 & \\
\hline & Lens & & 0.017 \\
\hline & Herat & & 0.017 \\
\hline & Pancreas & & 0.017 \\
\hline & Spleen & & 0.017 \\
\hline & Kidney & & 0.017 \\
\hline & Small intestine & & 0.017 \\
\hline & Prostate (male)/uteru & (female) & 0.017 \\
\hline Total & & 1.00 & 1.000 \\
\hline
\end{tabular}

\subsection{Evaluation of the PNDE}

PNDE were determined for 16 critical organs and remainders recommended by ICRP 103 as published [12]. In addition, prostate of male and uterus of female selected as at particular risk of fatal secondary malignancy recommended by ICRP 103 [11,13]. Table 2 lists the positions of 44 groups imparted within these phantoms and weighting factors $\left(W_{T}\right)$ [5]. The doses listed for the gonads and testes, as well as for the uterus and ovaries, were administered to males and females, respectively.

\subsection{Evaluating $N D_{T}$}

The PNDE was obtained for the position of chest PA. Meanwhile, PNDE denotes the total weighted equivalent doses of 16 organs and the "remainder" recommended by ICRP 103 as listed in Table 2, expressed as follows:

$$
P N D E=\sum_{T} W_{T} \cdot H_{T}
$$




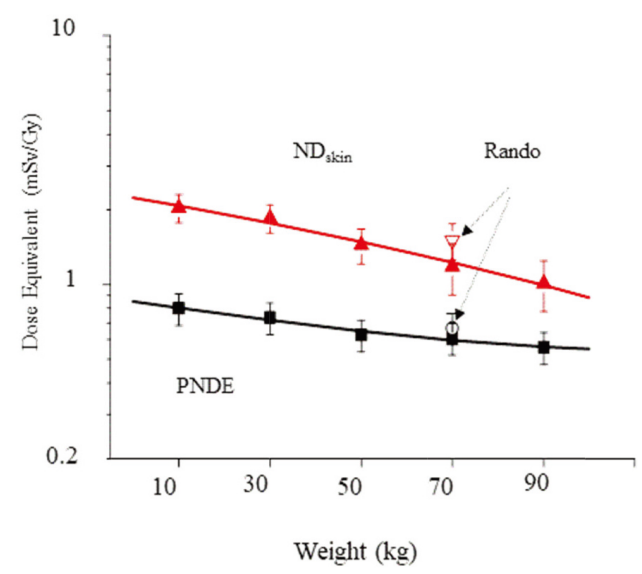

Fig. 2. Estimate of photoneutron dose equivalent (PNDE) and skin dose per Gy (mSv/Gy) undergoing lung cancer of $15 \mathrm{MV}$ IMRT treatments.

$H_{T}$ (equivalent dose), is multiplied by $W_{R}$, and obtained by

$$
H_{T}=\sum_{R} W_{R} \cdot N D_{T}
$$

Where $N D_{T}$ is the averaged ND of the organs. PNDE is in $\mathrm{J} / \mathrm{kg}$ unit and thus is termed Gray (Gy). Complete prescribed photon doses (200 cGy) of 15MV were delivered thus enabling detection of small ND outside the treatment field owing to radiation safety concerns. All measurements were conducted in triplicate for minimize fluctuations of TLDs. For small organs like the thyroid and gonad, $N D_{\text {thyroid }}$ and $N D_{\text {gonad }}$ obtained from the TLD-600 inserted in these organ centers served as the organ dose. For larger organs, like lung and colon, many TLDs were placed into each slices. The mean ND values were regarded as $N D_{\text {lung }}$ as well as $N D_{\text {colon }}$ (cf. Table 2).

\section{Results}

\subsection{Calibration of TLD-600}

The response linearity of photon dose for paired TLDs was tested ranging from $\mu \mathrm{Sv}$ to $\mathrm{mSv}$. Results demonstrated a good linearity at CCH. The dose-response of TLD-700 is y $(\mathrm{mSv})=2.05+0.144 \times$ $S N_{700}^{\gamma}(\mathrm{nC}), R^{2}$ is equal to 0.99893 for TLD-600 as well as 0.9994 for TLD-700, respectively. From measurements to calculate paired TLDs response to BNCT neutron, $C F_{600}^{n}$ from Eq. (3), is equal to 1.167 $\times 10^{-4} \mathrm{mSv} / \mathrm{nC}$, herein. This is in agreement with Hsu [9].

\subsection{Photoneutron dose equivalent (PNDE)}

Specifically, no standardized whole-body phantoms exist for evaluating the PNDE of different weights. Thus, this investigation developed five phantoms to simulate different body-weights, from 10 to $90 \mathrm{~kg}$. As illustrated in Fig. 2, PNDE at the different weights decrease abruptly with body-weight. Meanwhile, the maximum $N D_{\text {skin }}$ measurements at the $15^{\text {th }}$ slice were $1.98 \pm 0.30,2.03 \pm 0.26,1.85 \pm 0.24,1.44 \pm$ $0.23,1.18 \pm 0.27$, and $1.01 \pm 0.23 \mathrm{mSv} / \mathrm{Gy}$ for the Rando and PMMA phantoms, respectively. Fitting an 
exponential function (Eq. (6)) to this trend yielded the PNDE and $N D_{\text {skin }}$ 's relationship. Furthermore, these values reduced exponentially with increasing body-weight.

$$
\begin{aligned}
& P N D E=0.85-0.00519 M+2.15 \times 10^{-5} M^{2}, R^{2}=0.97493 \\
& N D_{\text {skin }}=2.23-0.0166 M+3.04 \times 10^{-5} M^{2}, R^{2}=0.98265
\end{aligned}
$$

Where PNDE and $N D_{\text {skin }}$ were administered in $\mathrm{mSv} / \mathrm{Gy}$, while M denotes PMMA phantoms in $\mathrm{kg}$ with $R^{2}$ is coefficient of determination.

\section{Discussions}

\subsection{Estimates of the secondary $N D_{T}$ of other organs}

Most neutrons those originate in the gentry head, then move from the tumor center to the patients lens and gonad during IMRT. Due to the ND of the 44 measurement (Table 2), estimates were evaluated that reflected the ND to anywhere in bodies of different body-weights for lung cancer treatment. Figure $3 \mathrm{a}-\mathrm{g}$ present the estimates of the total $N D_{T}$ on the distance away from the five phantoms' lung edge. Estimated $N D_{T}$ decreased with increasing distance from central axis. This phenomenon is also the same as the results published by Chao et al. and Lin et al. [2,6]. Comparisons with the measured values of these PMMA phantoms revealed that the small differences identified can be explained using seven different angles of multiple field incident these phantoms [2,6].

Measured $N D_{\text {liver }}$ differed among different points of the liver inside the Rando and PMMA phantoms. This study measured $N D_{\text {liver }}$ at the $22^{\text {nd }}$ slice of the phantoms. The averaged $N D_{\text {liver }}$ was $1.05 \pm 0.20$, and ranged from $1.24 \pm 0.21$ to $0.81 \pm 0.19 \mathrm{mSv} / \mathrm{Gy}$ of PMMA phantoms. The $N D_{\text {tumor }}$ of Rando was $2.70 \mathrm{mSv} / \mathrm{Gy}, 0.135 \%$ of $200 \mathrm{cGy}$ prescribed to PTV at the tumor center. Generally, in medical radiation exposure, patients are most worried about the gonads. Gonads are much more sensitive to radiation [7]. Averaged $N D_{\text {gonad }}$ obtained in this study was $0.25 \pm 0.04 \mathrm{mSv} / \mathrm{Gy}$, and ranged from $0.19 \pm 0.03$ to 0.32 $\pm 0.05 \mathrm{mSv} / \mathrm{Gy}$ (cf Fig. 2). The $N D_{\text {lung }}$ for lung exposed to $15 \mathrm{MV}$ stated large values, up to $1.02 \pm 0.13$ $\mathrm{mSv} / \mathrm{Gy}$ of lung cancer herein. $1.23 \pm 0.16(10 \mathrm{~kg}), 1.12 \pm 0.24(30 \mathrm{~kg}), 1.08 \pm 0.18(50 \mathrm{~kg}), 0.98 \pm$ $0.21(70 \mathrm{~kg}), 0.96 \pm 0.20(90 \mathrm{~kg}) \mathrm{mSv} / \mathrm{Gy}$ for these PMMA phantoms. Thyroid, red bone marrow, and remainder were comparatively low $N D_{T}$ during IMRT. The averaged PNDEs were $0.67 \pm 0.10$ and 0.71 $\pm 0.12 \mathrm{mSv} / \mathrm{Gy}$ for male and female of Rando phantoms, respectively. The averaged $N D_{\text {tumor }}$ were 2.70 $\pm 0.40,2.23 \pm 0.38,1.98 \pm 0.36,1.87 \pm 0.32,1.12 \pm 0.20$, and $0.92 \pm 0.17 \mathrm{mSv} / \mathrm{Gy}$ for Rando and 10 to $90 \mathrm{~kg}$ PMMA phantoms, respectively. $N D_{\text {lung }}$ at the lung center decreased with increasing distance to the tumor center (cf Fig. 3). Therefore, at the end of a $200 \mathrm{cGy}$ lung cancer treatment, Rando's PNDE was $0.67 \pm 0.10 \mathrm{mSv}$. PNDE from the $10 \mathrm{~kg}$ phantom was approximately $1.43 \pm 0.21$ times more than that of the $90 \mathrm{~kg}$ phantom.

\subsection{Comparison with other experimental data}

This is the first to use different body-weight of PMMA to evaluate PNDE herein, and compared the results presented here under lung cancer treatment with organ absorbed ND investigated with bubble detector in water used for $15 \mathrm{MV}$ x-ray linac-based radiotherapy of Siemens Primus, CCH under a 10 $\times 10 \mathrm{~cm}^{2}[1,6]$. The effective doses of fast and thermal neutrons on the water phantom surface were $1.42 \mathrm{mSv} / \mathrm{Gy}$, and thus were approximately 1.21 times higher than that of $70 \mathrm{~kg}$ phantom. These above differences can be attributed to different treatment planning, and phantom compositions of PMMA, epoxy-resin and water used for the conversion of absorbed dose. 
Table 3

PNDE (mSv/Gy) and estimated risk (\%) of secondary cancer coming from a total 72 Gy for Rando and PMMA phantoms

\begin{tabular}{ccc}
\hline Phantom $(\mathrm{kg})$ & PNDE $(\mathrm{mSv} / \mathrm{Gy})$ & Risk $(\%)$ \\
\hline Rando & $0.67 \pm 0.10$ & 2.85 \\
10 & $0.80 \pm 0.12$ & 8.35 \\
30 & $0.74 \pm 0.11$ & 7.73 \\
50 & $0.63 \pm 0.09$ & 2.68 \\
70 & $0.61 \pm 0.09$ & 2.59 \\
90 & $0.56 \pm 0.08$ & 2.38 \\
\hline
\end{tabular}

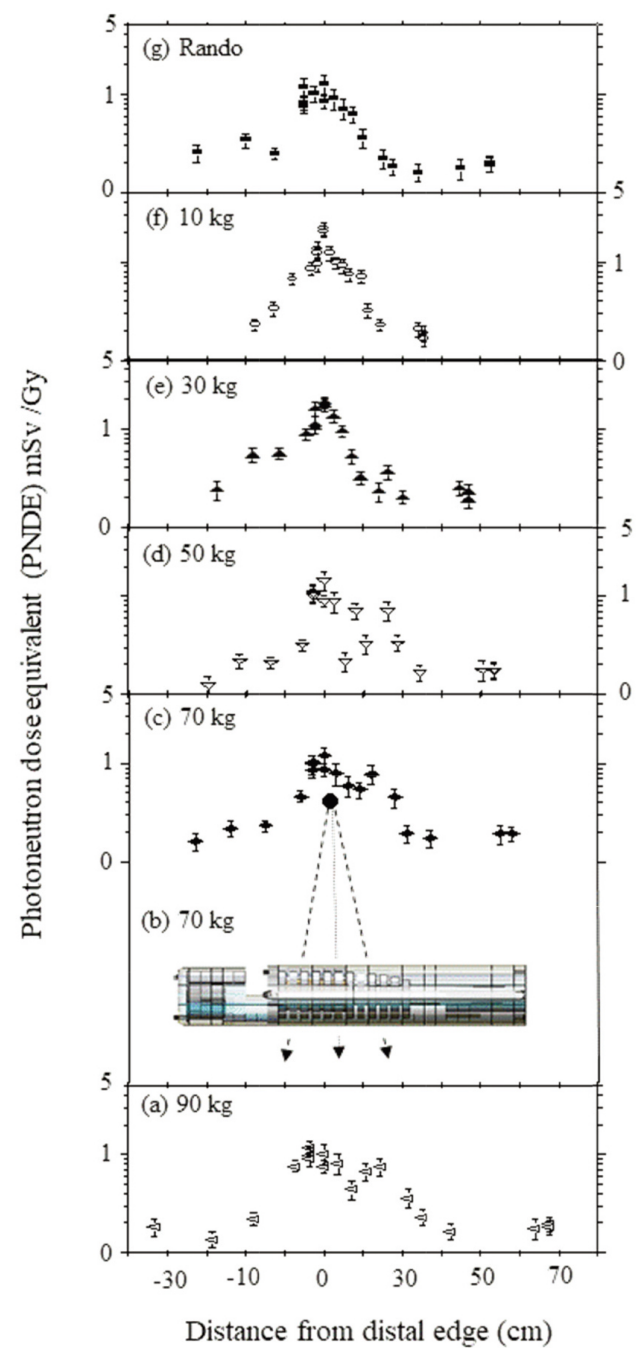

Fig. 3. Secondary ND at distal edge of lung on the central axis varying distances during IMRT treatment with $15 \mathrm{MV}$ (a) $90 \mathrm{~kg}$, (b) $70 \mathrm{~kg}$ irradiation, (c) $70 \mathrm{~kg}$, (d) $50 \mathrm{~kg}$, (e) $30 \mathrm{~kg}$, (f) $10 \mathrm{~kg}$, (g) Rando phantoms. 


\subsection{Risk of secondary malignancies}

The risk coming from neutron-induced malignancies is evaluated based on the differences between in the effective dose of the PMMA and Rando phantoms calculated by the BEIR VII [14]. BEIR VII stated that radiotherapy patients' risk should be approximately $5.9 \%$ per Sv for lung cancer patients given a large population spanning all ages and $14.5 \%$ per Sv for 10 year old male $[4,14]$. The prescription used for phantoms was 72 Gy of the PTV during IMRT. Comparing calculated PNDE of five PMMA phantoms, Table 3 demonstrates that the risk also increases with decreasing body-weight, as well as with decreasing PNDE. This study is in good agreement with Kry et al. stated the risk following prostate radiotherapy was $2-5 \%$ for all linac [15].

\section{Conclusion}

This feasibility study involves the evaluated PNDE of weight-dose relationship for each patient in the lung cancer treatment based on photoneutron contamination using the paired TLDs approach. The highest $N D_{T}$ was close to the tumor, and decreased with increasing distance from the tumor center. PNDE reduced with increasing phantom weight. Evaluated PNDE ranged from $0.80 \pm 0.12$ to 0.56 $\pm 0.08 \mathrm{mSv} / \mathrm{Gy}$ for these phantoms. The calculated PNDEs clearly show that the risk decreased with increasing body-weight. Results demonstrated that paired TLDs method exhibits high stability as well as sensitivity while evaluating PNDE.

High $N D_{\text {lung }}$ values were up to $1.02 \pm 0.13 \mathrm{mSv} / \mathrm{Gy}$ of Rando. To prevent unnecessarily extra ND to patients as well as satisfy the ALARA principle are strongly recommended. The in vivo $N D_{T}$ measurements reported in this study reveal a measurable difference in these PMMA phantoms that should be considered in cases involving extra secondary radiation. However, the risk also increases with decreasing body-weight and found the extra PNDE to be negligible.

\section{Acknowledgments}

The authors would like to thank senior radiotherapist Sing-Sheng Huang for many fruitful discussions, and radiotherapists of $\mathrm{CCH}$ and the staff of NTHU for their efficient support.

\section{Conflict of interest}

None to report.

\section{References}

[1] Hashemi SM, Hashemi-Malayeri B, Raisali G, et al. Measurement of photoneutron dose produced by wedge filters of a high energy linac using polycarbonate films. Journal of Radiation Research. 2008; 49(3): 279-283.

[2] Chao JH, Liu WS, Chen CY. Estimation of ${ }^{41} \mathrm{Ar}$ concentrations in the vicinity of a high-energy medical accelerator. Radiatation Measurments. 2007; 42: 1538-1544.

[3] National Council on Radiation Protection and Measurements, (1984) Neutron contamination from medical accelerators, NCRP-79. Washington, DC. 
[4] Harrison RM. Introduction to dosimetry and risk estimation of second cancer induction following radiotherapy. Radiation Measurements. 2013; 57: 1-8.

[5] Lin JP, Chua TC, Lin SY, et al. The measurement of photoneutrons in the vicinity of a Siemens Primus linear accelerator. Applied Radiation and Isotopes. 2001; 55: 315-321.

[6] Lin JP, Liu WC, Lin CC, Investigation of photoneutron dose equivalent from high-energy photons in radiotherapy. Applied Radiation and Isotopes. 2007; 65: 599-604.

[7] International Commission on Radiological Protection. (1991) Recommendation of the ICRP, ICRP Publication 60, Annals of the ICRP, No. 1-3; Pergamon Press, Oxford, UK.

[8] Ministry of Health and Welfare, (2019) Statistics of General Health Welfare of 2019, available at https://dep.mohw.gov.tw/ DOS/mp-113.html.

[9] Hsu FY, Chiu MC, Chang YL, et al. Estimation of photon and neutron dose distributions in the THOR BNCT treatment room using dual TLD method. Radiation Measurements. 2008; 43(2-6): 1089-1094.

[10] International Commission on Radiation Units and Measurement, (1992) Phantoms and computational models in therapy, diagnosis and protection ICRU-48. Bethesda.

[11] Matsumoto T. Monte Carlo simulation of depth-dose distribution in several organic models for boron neutron capture therapy. Nuclear Instruments and Methods in Physics Research, A. 2007; 508: 552-557.

[12] Lin HC, Lai TJ, Tseng HC, et al. Radiation doses with various body weights of phantom in brain 128-slice MDCT Examination. J Radiation Research. 2019; 60(4): 466-475.

[13] International Commission on Radiological Protection, (2007) Recommendations of the International Commission on Radiological Protection. ICRP-103, Pergamon Press, Oxford.

[14] Health Risks from Exposure to Low Levels of Ionizing Radiation, (2006) BEIR VII-Phase 2. Committee to Assess Health Risks from Exposure to Low Levels of Ionizing Radiation. National Research Council The National Academies Press, Washington DC.

[15] Kry SF, Salehpour M, Followill DS, et al. The calculated risk of fatal secondary malignancies from intensity-modulated radiation therapy. International Journal of Radiation Oncology Biology Physics. 2005; 62(4): 1195-1203. 